\title{
Erratum to: New Treatment Modalities for Hepatocellular Cancer
}

\author{
Kurt Mauer $^{1} \cdot$ Ryan O'Kelley $^{1} \cdot$ Nishant Poddar $^{2} \cdot$ Siobhan Flanagan $^{3}$. \\ Sameer Gadani ${ }^{1}$
}

Published online: 1 December 2015

(C) Springer Science+Business Media New York 2015

\section{Erratum to: Curr Gastroenterol Rep (2015) 17: 19 DOI 10.1007/s11894-015-0442-4}

The original version of this article unfortunately contained mistakes. Correct information is presented as follows.

(1) The name of the third author should have been displayed as "Nishant Poddar" (as shown above), not "Nishant Podda".

(2) Under the heading "RFA Versus Surgical Resection",

- Third paragraph, the sentence:

"Among the strengths of RFA in the treatment of HCC is the relatively low mortality and morbidity profile, with three recent multi-center studies have concluding mortal-

The online version of the original article can be found at http://dx.doi.org/ $10.1007 / \mathrm{s} 11894-015-0442-4$.

Sameer Gadani

gadanisd@slu.edu

1 Division of Interventional Radiology, Department of Radiology, Saint Louis University School of Medicine, 3635 Vista Ave at Grand Blvd, St Louis, MO 63110, USA

2 Division of Hematology and Oncology, Department of Internal Medicine, West Pavilion Cancer Center, 3655 Vista Ave, St. Louis, MO 63110, USA

3 Division of Interventional Radiology, Department of Radiology, University of Minnesota Medical Center, 420 Delaware St SE, Minneapolis, MN 55455, USA ity rates of less than $0.5 \%$ as well as major complication rates of 2.0 to $3.5 \%$ and minor complication rates of 5 to $8.9 \%$."

\section{SHOULD READ}

"Among the strengths of RFA in the treatment of HCC is the relatively low mortality and morbidity profile, with three recent multi-center studies have concluded mortality rates of less than $0.5 \%$ as well as major and minor complication rates of $2.0-3.5 \%$ and 5-8.9\% respectively."

- Fourth paragraph, the sentence:

"For very early-stage HCC, our preference is RFA when technically feasible."

\section{SHOULD READ}

"For very early-stage HCC, our preference is RFA / MWA when technically feasible."

(3) Under the heading, "Early-Stage HCC", the sentence:

"Similar to very early-stage HCC, the choice of therapy in terms of RFAversus surgical resection is controversial with RCTs reporting conflicting outcomes, rendering it difficult to draw conclusions regarding the optimal choice of therapy."

\section{SHOULD READ}

"In contradiction to the very early stage HCC where RFA / MWA is the preferred treatment, the choice of therapy in the early stage $\mathrm{HCC}$ in terms of RFA versus surgical 
resection is controversial with RCTs reporting conflicting outcomes.

(4) The heading "Combination Therapy_DEB-TACE and RFA Versus Monotherapy With RFA" SHOULD READ "Combination Therapy (DEB-TACE and RFA) Versus Monotherapy With RFA".

(5) The heading captured as "Very Early-Stage HCC, EarlyStage HCC, Intermediate-Stage HCC, Advanced-Stage HCC" SHOULD READ "Newer Percutaneous Ablation Therapies" and it is a sub-heading to Early-Stage HCC.

(6) Under the heading "Intermediate-Stage HCC", the sentence:

"Treatment options for this stage include transarterial therapies, including conventional transarterial chemoembolization (cTACE), transarterial chemoembolization with drug-eluting beads (DEB-TACE), embolization (TAE) [27], and radioembolization (TARE) and in certain circumstances, combination therapy $[3 \bullet \bullet] . "$

\section{SHOULD READ}

"Treatment options for this stage include transarterial therapies, including conventional transarterial chemoembolization (cTACE), transarterial chemoembolization with drug-eluting beads (DEB-TACE), bland embolization (TAE) [27], and transarterial radioembolization (TARE) and in certain circumstances, combination therapy $[3 \cdot \bullet] . "$
(7) In Table 1, the following changes are made.

- In row 1, in the column with the heading Tumor characteristics:

" $<2 \mathrm{~cm}$ or single $\mathrm{HCC}$ or 3 nodules less than $3 \mathrm{~cm}$ "

\section{SHOULD READ}

" $<2 \mathrm{~cm}$ (very early) or single HCC / 3 nodules less than $3 \mathrm{~cm}$ (early)".

- In row 1 , in the column with the heading Available treatment options:

"RFA, surgical resection"

SHOULD READ

"RFA / MWA, surgical resection"

- In row 3, in the column with the heading Available treatment options:

"RFA, MWA, combination therapy"

SHOULD READ

"RFA / MWA, combination therapy"

- Table 1 footnote: "MWA Microwave Ablation" is added. 